\title{
MULTI-LEVEL CONTROL OF AN INTELLIGENT WHEELCHAIR IN A HOSPITAL ENVIRONMENT USING A CYBER-MOUSE SIMULATION SYSTEM
}

\author{
Rodrigo A. M. Braga, Marcelo Petry, Eugenio Oliveira and Luis Paulo Reis \\ Artificial Intelligence and Computer Science Lab-LIACC, Faculty of Engineering of University of Porto \\ Rua Dr. Roberto Frias, $s / n$ 4200-465, Porto, Portugal \\ rodrigo.braga@fe.up.pt,marcelo.petry@gmail.com,eco@fe.up.pt,lpreis@fe.up.pt
}

Keywords: Intelligent Wheelchair, Intelligent Robotics, Strips Planning, Path Planning, A* Algorithm.

\begin{abstract}
The development of intelligent wheelchairs is a very good solution to assist severely handicapped people who are unable to operate classical electrical wheelchair by themselves in their daily activities. This paper describes the integration of a robotic simulator with our intelligent wheelchair shared control and planning modules. An adapted version of the free Cyber-Mouse robotic simulator was used to simulate the movement of the intelligent wheelchair in a hospital environment. Adaptations of the subsumption architecture, Strips Planning and $\mathrm{A}^{*}$ Algorithms were employed and integrated to allow wheelchair intelligent behavior. The experimental results have demonstrated the success of the integration of these algorithms in our simulator allowing very safe motion of the intelligent wheelchair in the simulated hospital environment. Also, the adapted Cyber-Mouse simulator proved its capability and robustness in simulating the hospital environment and wheelchair physic characteristics.
\end{abstract}

\section{INTRODUCTION}

Wheelchairs are important locomotion devices for handicapped and senior people. With the increase in the number of senior citizens and the increment of people bearing physical deficiencies in the social activities, there is a growing demand for safety and comfortable Intelligent Wheelchairs (IW) to practical uses. The main functions of IWs are (Faria, 2007a)(Faria, 2007b)(Jia, 2005):

-Interaction with the user, including hand based control, such as, joystick, keyboard, mouse, touch screen; voice based control, such as audio; vision based control, such as camera; and other sensor based control, such as pressure sensors.

-Afford Services, for instance autonomous navigation (with safety, flexibility and robust obstacle avoidance), communication with other devices (like automatic doors).

This paper discusses the application of a CyberMouse simulator, developed at the Univ.Aveiro in Portugal (Lau, 2002) in the study, development and test of shared control and high-level planning algorithms applied in an IW operating in a hospital environment.

A shared control algorithm was tested, allowing IW automatically avoids danger situations. Also, typical algorithms used in most intelligent robotics applications were applied in the control of the IW and simulated in the hospital scenery. Blended with the control, a motion planner was developed capable of generating the behavior/path commands according to an a-priori map of the world. This motion planner is capable of instructing the lowlevel motion controller module to achieve the highlevel commands desired by the user (Luo, 1999).

Cyber-Mouse is a competition among virtual robots, which takes place in a simulated environment running in a computer network. The simulation system creates a virtual arena with a starting grid, a target area, signalled by a beacon, and populated with obstacles. It also creates the virtual bodies of the robots. The simulator estimates sensor measures which are sent to the agents. Reversely, receives and apply actuating orders coming from agents (Lau, 2002).

The paper is subdivided in 5 different sections: section 2 presents some brief definitions of the control algorithms applied in this research; section 3 contains a description of the system developed; section 4 provides experimental tests and result discussion and section 5 presents the final conclusions and points out some future research topics. 


\section{INTELLIGENT WHEELCHAIR CONTROL ALGORITHMS}

To test the simulator, the algorithms used to implement the control system and algorithms used for the high-level planning system were:

- Adapted Subsumption Architecture. (Brooks, 1991) (Russell, 2002)(Ferber, 1991) for basic moving;

- Knowledge Representation using Grids. Spatial knowledge for robotic movement, navigation and planning (Borenstein, 1991)(Thrun, 1996).

- A* Search Algorithm for Trajectory Generation. used to find a path from a given initial node to a given goal node (Shapiro, 2000) (Hart, 1968) (Barr, 1986).

- Adapted Strips Planning Algorithm. Planning is the task of searching for an action sequence to achieve a given goal (Russell, 2002)(Weld, 1999)(Fikes, 1971) (Bonet, 2001).

\section{SYSTEM DESCRIPTION}

The hospital environment was created modifying the Cyber-Mouse labyrinth definition XML file that contains positions of a set of walls of arbitrary shape and beacons that emit signals. Figure 1 shows our hospital modified floor plan.

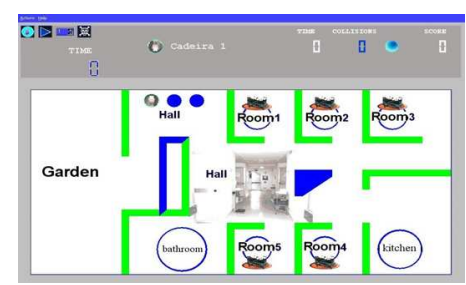

Figure 1: Hospital environment represented using the Cyber-Mouse simulator.

The hospital representation is composed of five rooms: bathroom, kitchen, hall and garden and a set of walls of different shapes. IWs are represented by modified robotic agents. UDP Sockets are used for communication between the robotics agents and the simulator. The communication is based in dispatching XML messages of five types: request register; refuse reply; acceptation reply; sensor data, action order.

Figure 2 presents the developed agent control software interface. A communication configuration panel can be seen in left side of the interface, the sensor values are depicted in the middle of the interface. In the right side, there are three function modules. The first module has the following functions: semi-automatic control, revolve in a point direction, move to a point, move through planning path.

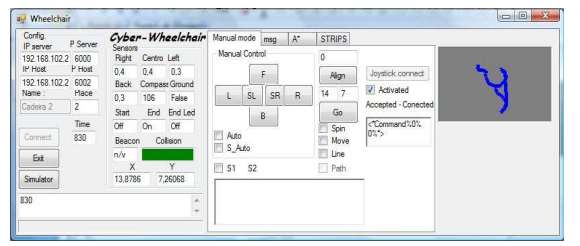

Figure 2: Intelligent Wheelchair software interface.

The semi-automatic control, also denoted shared control, was constructed with fusion between user command and subsumption architecture. This way it achieves safe movement allowing automatic obstacle avoidance. Figure 3 presents the implemented architecture.

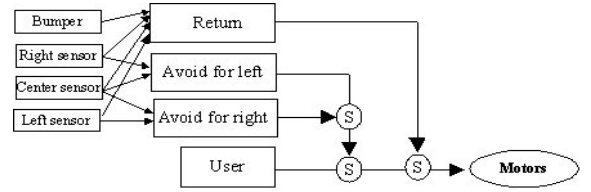

Figure 3: Subsumption architecture used in shared control.

Figure 4 shows the path generation interface module. The path generation was implemented using the $A^{*}$ algorithm. The state world information is loaded into the system using a XML file. A grid of $56 \times 28$ cells representing the world- state, for this development, was considered as the world state environment. Also, object extension techniques were used, in such manner that a robot may be represented as a point in the system without the risk of collision with the walls.

The path generation module searches for a path, starting in the actual point and finishing in the user selected point. The path generated can be seen in the interface provided.

The last module (see Figure 5) is an automatic planning module developed based in Strips Planning Algorithm with inverse chaining. Initially, we developed five general high-level objectives: "gobathroom", "go-garden", "go-room", "gokitchen", "go-consulting room". These general objectives generate different final objectives, depending on the patient, wheelchair and location set for the actions. For example, the action patient 1 with wheelchair 2 go-room for Room 1 results in the objective: patient 1 in Room 1, patient 1 without wheelchair, wheelchair 2 free and situated in hall. In this way, it is allowed to set many different final objectives and since the plan depends on the initial 
state, it will have different sequences and number of actions for arriving the final objective.

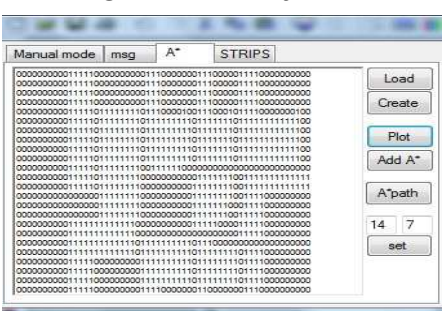

Figure 4: Path generation module interface.

After plan generation, we can execute it in the simulated environment. Now, the system uses the path generation module for the generation the paths for movement actions and automatic move control module to execute the movement through the path generated with collision avoidance. This module executes step by step the actions of the plan using the other modules whenever it is necessary.

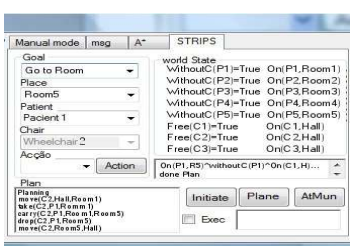

Figure 5: Planning module interface.

\section{EXPERIMENTS AND RESULTS}

This section presents a simple operation example of our robotic agent and results gathered in this experiment. After initiating the simulator, the configuration, map and grid file are loaded in the simulator, through a XML file.

As focus is primarily testing the simulation system and the implementation of an ordinary multi level control, sensor and motor noise were disable. Another simplification is the robot data position, which is provided by the simulator GPS system that represents a robust odometry system.

The simulator configuration is presented in Table 1. The simulator allows inserting noise in the robots sensors and actuators. However in this stage of development, the sensor and actuators noise was not used.

The first test was the use of the $A^{*}$ algorithm for searching the path from point $(x=9,5$ and $y=13,2)$ to point $(x=14$ and $y=7)$. The plan devised is presented in Table 3. The plan is presented in inverse order, i.e., the first point is in the table bottom and last point is in the table top.
Table 1: XML file to configure simulator.

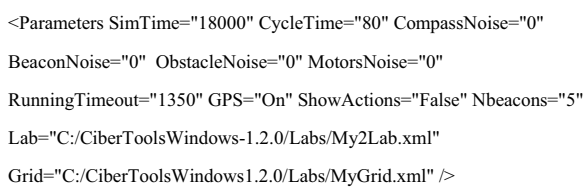

The resulting movements are shown in Figure 6. The black letters represent before of move, the blue line represent plan path and the red line represent the path travelled. The number ' 0 ' represents free space, ' 1 ' represents the expanded wall. In this test, the move was free collision and with minimum trajectory error.

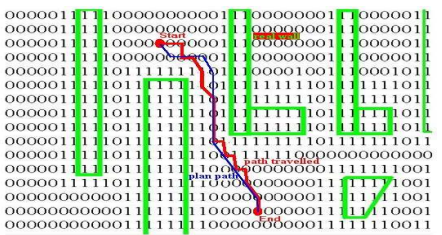

Figure 6: Movement result.

Next step was to generate a comprehensive plan using the implemented path generator. The goal in this case was to pick up patient 1 in bedroom 1 ; carry him to bedroom 5 , to make company to patient 5; and finally go back to the lobby. In this example we had as objective: "Go to the bedroom", as Place: 5 and Patient: 1. This parameters result as final objective: $\operatorname{On}(P 1, R 5) \wedge$ WithoutC $(P 1) \wedge$ On $(C 2, H)$. The world state before the action, the resulting plan and the following state are represented in Figure 7.
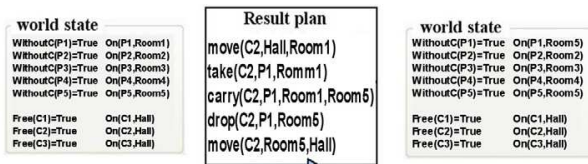

Figure 7: World state s, plan and world state s'.

The grid containing the final state and the travelled path, based on the plan mentioned before, can be observed in Figure 8. This grid contains the four basic movements that the IW needed to perform to achieve the final objective: from the initial point until the lobby, from lobby to bedroom 1 (to pick up patient 1), then to the bedroom 5 and after leave patient 1 going back (empty) to the lobby. The time to conclude movement in automatic control was 982 second and for manual control was 870 seconds.

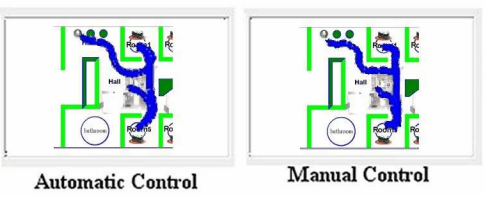

Figure 8: Final movement result. 


\section{CONCLUSIONS}

This paper presented the implementation of a robotic Intelligent Wheelchair, simulating its behavior in an adapted Cyber-Mouse simulator. This agent resulted of a modular system composed of three modules. The first one is a shared control that merges user commands with the information received by the IW sensors. This advantage prevents the collision with objects in its way becoming movement system more reliable and safe. The algorithms developed use a subsumption architecture: once there are objects close to the wheelchair, the user commands are disabled and deflect commands are sent to the motors, ensuring a safe motion.

A module was develop using the $\mathrm{A}^{*}$ algorithm as path generator to calculate the shortest path from the robot's actual position to the objective point. The third module was implemented to plan some usual tasks in a hospital environment, using Strips planning to solve these interactions. In most of the cases Strips proved to be efficient, delivering complete plans, with exceptions presented under Sussman anomaly effects, where one operation cancels other in the plan.

It was also demonstrated that Cyber-Mouse is a friendly tool to test control algorithms, IW navigation and its interaction with hospital environment.

Future research directions include the improvement to non-linear planning and upgrade from $A^{*}$ to $D^{*}$ algorithm, once it's preferable in such a dynamic environment. To be fully intelligent, it's not enough the wheelchair to plan its own path or share its control. It is also necessary to communicate with other intelligent wheelchairs and devices like doors activation systems, elevators and lights. Due to this, it is intended to perform an in depth study in proper methodologies to implement these capacities in the wheelchair, and this way, implement some functions as cooperative behavior among a group of IW and collaboration among the user and the system. In Cyber-Mouse it is necessary to increase its present simulation capacities, from its actual three IW, enabling hybrid systems test, where real and virtual IW interact with each other. These interactions make possible high complexity tests with a substantial number of devices and wheelchairs, representing a reduction in the project costs, once there wouldn't be necessary a large number of real IW. Still, in Cyber-Mouse, it is necessary to implement noise treatment in the motors and sensors to have actions in the simulated wheelchair closer to those of real wheelchairs.

\section{REFERENCES}

Barr, A. Cohen, P. Feigenbaum, E., 1986. Handbook of Artificial Intelligence. Addison Wesley. Vol. 1.

Bonet, B. and Geffner, H., 2001. Heuristic search planner 2.0. The AI Magazine, 22(1), pp.77-80.

Borenstein, J., Koren, Y., 1991. The Vector Field Histogram - Fast Obstacle Avoidance for Mobile Robots, IEEE Journal of Robotic and Automation, v. 7, n. 3, pp. 278-288.

Brooks, R. A., 1991. Intelligence Without Reason. Proc. 12th Int. Joint Conference on Artificial Intelligence IJCAI-91, Sydney, Australia. pp.569-595.

Faria, P. M., Braga, R A. M., Valgôde, E., Reis L. P., 2007a. Platform to Drive an IW using Facial Expressions. Proc. 9th Int. Conf. on Enterprise Information Systems, HCI, ICEIS'2007. pp.164-169. Funchal, Portugal. June 12-16.

Faria, P. M., Braga, R. A. M., Valgôde, E., Reis, L. P., 2007b. Interface Framework to Drive an Intelligent Wheelchair Using Facial Expressions. IEEE Int. Symp. on Industrial Electronics, pp. 1791-1796, June 4-7.

Ferber, J.; Gasser, L., 1991. Intelligence artificielle distribuée. In: Int. WorkShop on Expert Systems \& Their Applications, 10., Avignon. Cours n. 9. France.

Fikes, R., Nilsson, N. J., 1971. STRIPS: A New Approach to the Application of Theorem Proving to Problem Solving. IJCAI 1971. pp.608-620.

Hart, P. E., Nilsson, N. J. and Bertram R., 1968, A formal basis for the heuristic determination of minimum cost paths. IEEE Transactions on Systems Science and Cybernetics, 4(2):pp.100-107.

Jia, P. and Hu, H., 2005. Head Gesture based Control of an Intelligent Wheelchair. CACSUK- $11^{\text {th }}$ Ann. Conf. Chinese Aut. Comp. Society in the UK, Sheffield, UK.

Lau, N., Pereira, A., Melo, A., Neves, A. and Figueiredo, J., 2002. Ciber-Rato: Um Ambiente de Simulação de Robots Móveis e Autónomos. Rev. DETUA, Vol. 3, Nr. 7, pp.647-650, Sept.

Luo, R. C., Chen, T. M. and Lin, M. H., 1999. Automatic Guided IW System Using Hierarchical Grey-Fuzzy Motion Decision-Making Algorithms, Proc. IEEVRSJ 1999 Int. Conf. on Intelligent Robots and Systems.

Russell, S. and Norvig, P., 2002. Artificial Intelligence: A Modern Approach. Prentice Hall, E.Cliffs, NJ, $2^{\text {nd }}$ ed.

Shapiro S.C., 2000. Encyclopedia of Artificial Intelligence. Wiley-Interscience. May.

Thrun, S. and Bücken, A., 1996. Integrating Grid-Based and Topological Maps for Mobile Robot Navigation, In: Proc. 13th National Conference on Artificial Intelligence AAAI, Portland, Oregon, pp.944-950.

Weld, D. S., 1999. Recent advances in AI planning. AI Magazine, pp.93-123 